\title{
EXPLICIT CONSTRUCTION OF GROUP VARIETIES RELATED TO SOLITON SOLUTIONS OF THE KP HIERARCHY
}

\author{
FRANCESCO BOTTACIN
}

\begin{abstract}
A remarkable relation between $N$-soliton solutions of the KP hierarchy and some group varieties is found. Precisely, we prove that to each such solution there is associated, in a natural way, a commutative group variety, which we are able to construct explicitly. These varieties turn out to be products of multiplicative groups.
\end{abstract}

\section{INTRODUCTION}

In a previous paper [3] we have shown that to each element in a large class of solutions to the KP hierarchy there is associated, in a natural way, a group variety. This result was achieved by showing that these solutions are in fact functions of theta type. Let us recall here that the functions of theta type were introduced by I. BARSOTTI in 1968 as a generalization of classical theta functions: a (holomorphic) theta type is essentially a formal power series in $n$ indeterminates, $\theta(x) \in k[[x]]$, which satisfies the holomorphic prosthaferesis:

$$
\theta(x+y) \theta(x-y) \in k[[x]] \otimes_{k} k[[y]] .
$$

It turns out that the field generated over $k$ by the iterated logarithmic derivatives of a theta type $\theta(x)$, from the seconds on, is the function field of a commutative group variety, called the group variety of $\theta$. Needless to say, when $k$ is the complex field, the theory of theta types covers the classical theory of theta functions.

In this paper we restrict to a class of solutions of particular interest in physics, namely the so-called $N$-solitons. We prove that an $N$-soliton solution to the KP hierarchy is actually a holomorphic theta type, and its associated group variety is a product of multiplicative groups. Hence the same conclusions also hold for the $\mathrm{KdV}$ and all other hierarchies which can be obtained as specializations of the KP. Moreover all these constructions are made explicitly.

This fact sheds a new light on a result proved by D. MUMFord in [7]: he showed that starting from the Jacobian theta function of a nonsingular curve one can construct solutions to the KP hierarchy, and these functions also give solutions to the $\mathrm{KdV}$ hierarchy when the curve is taken to be hyperelliptic. Moreover, if one takes an algebraic family $C_{t}$ of smooth hyperelliptic curves of genus $g$, which, as $t \rightarrow 0$, tend to a singular curve $C_{0}$ having only nodes as singularities, then the Jacobian varieties Jac $C_{t}$ of the curves tend to a generalized Jacobian Jac $C_{0}$, which is precisely 
$\left(k^{*}\right)^{g}$, the product of $g$ copies of the multiplicative group $k^{*}$. Under this deformation, Riemann's theta functions on the abelian varieties Jac $C_{t}$ tend to a function on the generalized Jacobian Jac $C_{0}$ : this is the analogue of the classical Riemann theta function on a group variety which is no longer abelian (it is what we call a theta type). The fundamental fact is that this function is essentially the $g$-soliton solution of the KdV hierarchy.

Our result shows that this is not a special case but only an aspect of a general situation: every soliton solution of the KP hierarchy is actually a theta type of a multiplicative group.

This paper is organized as follows: in Sect. 1 we recall the definitions and some basic results concerning functions of theta type. Then we also recall the definition of hyperfield and its connections with group varieties, essentially because this terminology, introduced by I. BARSOTTI, is not at all "well-known" to the mathematical public.

In Sect. 2 we first give a brief description of the KP hierarchy and recall how it can be expressed as a set of Hirota's bilinear differential equations for a $\tau$-function. Then we introduce infinite order differential operators, called vertex operators, which act on the space of $\tau$-functions, sending a given solution to the KP hierarchy to a new one. This allows us to construct the famous $N$-soliton $\tau$-function, obtained by letting a particular vertex operator act on the constant function 1 . Finally we describe in detail how one can find an explicit expression for the solitons constructed in this way.

In Sect. 3 we make use of the explicit expression previously found to prove that $N$-soliton $\tau$-functions are functions of theta type, and their associated group varieties are precisely the multiplicative groups. The last part of the Section is then devoted to the analysis of the dependence of the dimension of these varieties on the original soliton.

\section{Theta TYPES AND GROUP VARIETIES}

Let $k$ be an algebraically closed field of characteristic zero, and $k[[x]]=k\left[\left[x_{1}, \ldots, x_{n}\right]\right]$ denote the ring of formal power series in $n$ variables, with coefficients in $k$. For an integral domain $I$, the symbol $Q(I)$ denotes the quotient field of $I$.

A non-zero element $\theta(x) \in Q(k[[x]])$ is a function of theta type, or simply a theta type, if it satisfies the prosthaferesis:

$$
\theta(x+y) \theta(x-y) \in Q\left(k[[x]] \otimes_{k} k[[y]]\right) .
$$

A theta type $\theta(x)$ is holomorphic if it satisfies the holomorphic prosthaferesis (hence it belongs to $k[[x]])$ :

$$
\theta(x+y) \theta(x-y) \in k[[x]] \otimes_{k} k[[y]] .
$$

It follows that a theta type is always the quotient of two holomorphic theta types. It is not useless to remember here that, if $k$ is the complex field, a holomorphic theta 
type is actually an entire function, i.e. its power series converges, and the theory of theta types generalizes the classical theory of theta functions (this is proved in $[1$, Sect. 4]).

We need some more definitions: two theta types are associate if their ratio is a quadratic exponential, i.e. a factor of the form $a \cdot \exp q(x)$, where $a \in k$ and $q(x)$ is a polynomial of degree $\leq 2$, with vanishing constant term. If $\theta\left(x_{1}, \ldots, x_{n}\right)$ is a theta type, it may happen that there exist a $\theta^{\prime}$, associate to $\theta$, and linear combinations $y_{1}, \ldots, y_{m}$ of $x_{1}, \ldots, x_{n}$ with coefficients in $k$ and $m<n$, such that $\theta^{\prime}(x) \in Q(k[[y]])$. In other words $\theta$ itself, or one of its associates, may effectively depend on less then $n$ variables. The least $m$ for which this is possible is called the dimension of $\theta, \operatorname{dim} \theta$. It also equals $n-m^{\prime}$, where $m^{\prime}$ is the maximum, as $\theta^{\prime}$ varies among the associates of $\theta$, reached by the dimension of the $k$-vector space of derivations $d \in \operatorname{Der} Q(k[[x]])$ (i.e. linear combinations of the $d_{i}=\partial / \partial x_{i}$, with coefficients in $k$ ) that annihilate $\theta^{\prime}$. A theta type of dimension $<n$ is called degenerate.

Now we briefly recall the definition and basic properties of hyperfields, refering the reader to [1] and [6] for a more detailed treatment of this subject.

A hyperfield $C$ over $k$ is an extension field of $k$ which satisfies the following properties:

(i) $C \otimes_{k} C$ and $C \otimes_{k} C \otimes_{k} C$ are integral domains: this is true for all extensions $C$ of $k$, if $k$ is algebraically closed;

(ii) there is given a unitary $k$-algebra homomorphism $\mathbf{P}: C \rightarrow Q\left(C \otimes_{k} C\right)$, called the coproduct, which is coassociative (i.e. it satisfies $(\iota \otimes \mathbf{P}) \mathbf{P}=(\mathbf{P} \otimes \iota) \mathbf{P}$, where $\iota$ is the identity map on $C)$ and cocommutative $(\delta \circ \mathbf{P}=\mathbf{P}$, where $\delta$ is the natural extension to $Q\left(C \otimes_{k} C\right)$ of the map sending $x \otimes y \in C \otimes_{k} C$ to $y \otimes x)$

(iii) there is also given a unitary endomorphism $\rho$ of the $k$-algebra $C$, called the inversion, such that $\mu(\iota \otimes \rho) \mathbf{P} x=\epsilon x \in k$, for all $x \in C$ for which this expression makes sense, where $\mu$ is the natural extension to $Q\left(C \otimes_{k} C\right)$ of the multiplication map $C \otimes_{k} C \rightarrow C$ sending $x \otimes y$ to $x y$. The map $\epsilon$ defined by the preceding formula is called the coidentity;

(iv) $C$ is equal to the quotient field of the local ring $R_{C}=\epsilon^{-1} k$;

(v) $\mu(\iota \otimes \epsilon) \mathbf{P} x=x$, for all $x \in C$.

From these conditions it follows that $\mathbf{P}$ and $\rho$ are isomorphisms and $\rho^{2}=\iota$.

We also define the notion of subhyperfield: if $C$ and $K$ are two hyperfields over $k$, we say $C$ is a subhyperfield of $K$ if it is a subfield of $K$ and its coproduct and inversion are induced by those of $K$.

The basic example of hyperfield is constructed as follows: let $A$ be a commutative group variety over $k$, and $C=k(A)$, the function field of $A$. Take as the coproduct $\mathbf{P}: C=k(A) \rightarrow Q\left(C \otimes_{k} C\right)=k(A \times A)$ the natural map induced by the composition law on $A$, and as $\rho: C \rightarrow C$ the map induced by the morphism which sends a point 
in $A$ to its inverse, for the given group law (when this is defined). This $C$ turns out to be a finitely generated hyperfield over $k$.

The fundamental fact is that the converse is also true, i.e. for any finitely generated hyperfield $C$ over $k$, there exists a commutative group variety $A$ over $k$, such that $C=k(A)$, the composition law on $A$ being dual to the coproduct of $C$. In this situation we say that $A$ is the group variety of $C$, and $C$ is the hyperfield of $A$. Moreover, if $C=k(A)$ and $K=k(B)$ are hyperfields, for some group varieties $A$ and $B$, then $C$ is a subhyperfield of $K$ if and only if there is a surjective homomorphism of group varieties $B \rightarrow A$ corresponding to the inclusion of $C$ in $K$.

Now we come back to theta types. Given a theta type $\theta(x) \in Q(k[[x]])$, there is a natural way to associate to it a hyperfield $C \subset Q(k[[x]])$, whose coproduct and inversion are induced by those defined on $k[[x]]$ (which is not a hyperfield!):

$$
\begin{aligned}
\mathbf{P}: k[[x]] & \rightarrow k[[x]] \hat{\otimes} k[[x]] \cong k[[x, y]] \\
x_{i} & \mapsto x_{i} \hat{\otimes} 1+1 \hat{\otimes} x_{i}
\end{aligned}
$$

and

$$
\begin{gathered}
\rho: k[[x]] \rightarrow k[[x]] \\
x_{i} \mapsto-x_{i} .
\end{gathered}
$$

We don't mention here how this can be done (see [1] or [2]), but we recall that $C$ turns out to be finitely generated over $k$ by the iterated logarithmic derivatives of $\theta$, from the seconds on, hence it is the hyperfield of a commutative group variety $A$, called the group variety of $\theta$. It follows immediately that two associate theta types have the same hyperfield and group variety. Then we define the transcendency of $\theta$, $\operatorname{transc} \theta$, as $\operatorname{transc} C / k$, and it can be easily proved that $\operatorname{transc} \theta \geq \operatorname{dim} \theta ; \theta$ is called a theta function when equality holds.

To end this Section, we just mention that there is also a natural way to define a divisor on $A$, called the divisor of $\theta$, which is effective if and only if $\theta$ is holomorphic, and is linearly equivalent to zero if and only if $\theta \in C$ (modulo substitution of $\theta$ with an associate theta type). All this works well, in the sense that it gives a generalization of the classical notion of theta divisor. We refer to [1] and [2] for further information.

\section{THE KP HIERARCHY AND ITS SOLITON SOLUTIONS}

In this Section we briefly recall the definition of the Kadomtsev-Petviashvili (KP) hierarchy and its expression as a set of Hirota's bilinear differential equations for a $\tau$-function. Then we introduce the $N$-soliton solutions to the KP hierarchy and find their explicit expressions. For a detailed account of this subject the reader is referred to [4]. Let $k$ be the complex field and $x$ denote the infinite set of indeterminates $x_{1}, \ldots, x_{n}, \ldots$ For convenience of notation, we simply write $\partial$ for the derivative $\partial / \partial x_{1}$ 
A (formal) pseudodifferential operator $P$ is a formal Laurent series in $\partial^{-1}$, with coefficients in $k[[x]]: P=\sum_{j=-\infty}^{m} a_{j}(x) \partial^{j}$.

For a pseudodifferential operator $P$, we denote by $P_{+}=\sum_{j=0}^{m} a_{j}(x) \partial^{j}$ the ordinary differential operator part, and by $P_{-}=\sum_{j<0} a_{j}(x) \partial^{j}$ its residual part.

We also introduce the space of formal oscillating functions

$$
\left\{f(x, \lambda) \exp \xi(x, \lambda) \mid f(x, \lambda) \in k[[x]]\left(\left(\lambda^{-1}\right)\right)\right\},
$$

where $\lambda$ is a formal parameter and $\xi(x, \lambda)=\sum_{i=1}^{\infty} x_{i} \lambda^{i}$.

Let $w(x, \lambda)=\left(\sum_{i=0}^{\infty} w_{i}(x) \lambda^{-i}\right) \exp \xi(x, \lambda)$ be a formal oscillating function, normalized by letting $w_{0}(x)=1$, and consider the following system of linear equations:

$$
\left\{\begin{array}{l}
L w=\lambda w \\
\frac{\partial w}{\partial x_{n}}=B_{n} w \quad n=1,2, \ldots
\end{array}\right.
$$

where $L$ is a pseudodifferential operator of the form $L=L(x)=\partial+u_{-1}(x) \partial^{-1}+$ $u_{-2}(x) \partial^{-2}+\ldots$, and $B_{n}=\left(L^{n}\right)_{+}$.

The KP hierarchy is defined as the system of non-linear differential equations for the $u_{-i}(x)$ 's, resulting from the compatibility conditions of (2.1); these turn out to be equivalent to the system of Lax equations for the operator $L$ :

$$
\frac{\partial L}{\partial x_{n}}=\left[B_{n}, L\right], \quad n=1,2, \ldots
$$

Moreover it can be shown that the system (2.1) has a solution $w$ if and only if $L$ satisfies the KP hierarchy (2.2), in which case the solution is unique up to multiplication by elements in $1+k\left[\left[\lambda^{-1}\right]\right] \lambda^{-1}$ (see, for example, [8, Sect. 1.2]).

We must now make a brief digression to introduce Hirota's bilinear differential operators: for polynomials $P\left(y_{1}, \ldots, y_{n}\right) \in k\left[y_{1}, \ldots, y_{n}\right]$ and functions $f(x)$ and $g(x)$, they are defined as

$$
P\left(D_{1}, \ldots, D_{n}\right) f \cdot g=\left.P\left(\partial / \partial y_{1}, \ldots, \partial / \partial y_{n}\right)[f(x+y) g(x-y)]\right|_{y=0} .
$$

The differential equations $P(D) f \cdot g=0$ are Hirota's bilinear differential equations.

Returning to our previous situation, it is shown in [4, Sect. 1] that if $w(x, \lambda)$ is a solution of (2.1), for a pseudodifferential operator $L$ satisfying (2.2), then there exists a power series $\tau(x) \in k[[x]]$, called the $\tau$-function associated to $L$, such that

$$
w(x, \lambda)=\frac{\tau\left(x_{1}-\frac{1}{\lambda}, x_{2}-\frac{1}{2 \lambda^{2}}, x_{3}-\frac{1}{3 \lambda^{3}}, \ldots\right)}{\tau(x)} \exp \xi(x, \lambda) .
$$

Moreover the KP hierarchy can be written equivalently as an infinite set of Hirota's bilinear differential equations for the $\tau$-function $\tau(x)$, obtained from the following generating function expansion:

$$
\sum_{i=0}^{\infty} p_{i}(-2 y) p_{i+1}(\tilde{D}) \exp \left(\sum_{j=1}^{\infty} y_{j} D_{j}\right) \tau \cdot \tau=0,
$$


where $p_{n}(x)$ are Schur polynomials, defined by

$$
\exp \left(\sum_{j=1}^{\infty} x_{j} q^{j}\right)=\sum_{n=0}^{\infty} p_{n}(x) q^{n},
$$

and $\tilde{D}=\left(D_{1}, 2^{-1} D_{2}, 3^{-1} D_{3}, \ldots\right)$ with the $D_{j}$ 's being Hirota's symbols, so that every coefficient of (2.3), regarded as a power series in the $y$ 's, gives Hirota's equation for a $\tau$-function.

An important result states that the set of solutions to (2.3) admits the transformation $\tau(x) \rightarrow \exp (a X(p, q)) \tau(x)$, sending a given solution $\tau(x)$ to a new one. Here $a, p, q$ are parameters, and $X(p, q)$ is an infinite order differential operator, called a vertex operator, defined as follows:

$$
X(p, q)=\exp (\xi(x, p)-\xi(x, q)) \exp \left(-\xi\left(\tilde{\partial}, p^{-1}\right)+\xi\left(\tilde{\partial}, q^{-1}\right)\right),
$$

where $\tilde{\partial}=\left(\partial / \partial x_{1}, 2^{-1} \partial / \partial x_{2}, 3^{-1} \partial / \partial x_{3}, \ldots\right)$.

This result provides an effective method to construct explicit solutions to the KP hierarchy (2.3): just start with one known solution and let the operators $\exp \left(a_{i} X\left(p_{i}, q_{i}\right)\right)$ act repeatedly on it. In particular, starting from the constant function 1 (which obviously solves (2.3)!), we can get a special solution defined as follows:

$$
\tau(x)=\exp \left(\sum_{i=1}^{N} a_{i} X\left(p_{i}, q_{i}\right)\right) 1 .
$$

This is precisely the $N$-soliton solution of the KP hierarchy (actually the classical $N$-soliton is given by $\left.\partial^{2} / \partial x_{1}^{2} \log \tau(x)\right)$.

Now we want to describe in detail how one can find the explicit expression of the $N$-soliton $\tau$-function, starting from its definition (2.4). We divide what follows into 3 steps, whose proof consists of straightforward computations which are left to the reader.

Step 1. First observe that the operator $\exp (\lambda \partial / \partial t)$ acts by translation on $t$ :

$$
\exp (\lambda \partial / \partial t) f(t)=f(t+\lambda)
$$

from this it follows immediately that the action of the vertex operator $X(p, q)$ on a function of infinitely many variables is the following:

$$
X(p, q) f\left(x_{1}, x_{2}, \ldots\right)=\exp (\xi(x, p)-\xi(x, q)) f\left(\ldots, x_{i}-\frac{1}{i p^{i}}+\frac{1}{i q^{i}}, \ldots\right) .
$$

In particular we note that $X\left(p_{i}, q_{i}\right) 1=\exp \xi_{i}$, where we set $\xi_{i}=\xi\left(x, p_{i}\right)-\xi\left(x, q_{i}\right)$, and this allows us to evaluate the first order terms in the series expansion of (2.4).

Step 2. Note that from the power series expansion of

$$
\log \left(\frac{1}{1-\lambda}\right)=-\log (1-\lambda)=\sum_{i=1}^{\infty} \frac{\lambda^{i}}{i}
$$


we formally have: $\exp \left(\sum_{i=1}^{\infty} \frac{\lambda^{i}}{i}\right)=\frac{1}{1-\lambda}$.

Using this simple remark, we can evaluate $X\left(p_{k}, q_{k}\right) \exp \left(\sum_{i=1}^{n} \xi_{i}\right)$, and find that it is equal to $\prod_{i=1}^{n} c_{k i} \exp \left(\xi_{k}+\sum_{i=1}^{n} \xi_{i}\right)$, where we set

$$
c_{k i}=\frac{\left(p_{k}-p_{i}\right)\left(q_{k}-q_{i}\right)}{\left(p_{k}-q_{i}\right)\left(q_{k}-p_{i}\right)} .
$$

Finally, using induction on $m$, and noting that $c_{i j}=c_{j i}$ and $c_{i i}=0$, it is not difficult to prove the following result:

$$
\left[\sum_{i=1}^{N} a_{i} X\left(p_{i}, q_{i}\right)\right]^{m} \cdot 1=m ! \cdot \sum_{1 \leq i_{1}<\cdots<i_{m} \leq N} \prod_{j=1}^{m} a_{i_{j}} \cdot \prod_{1 \leq h<k \leq m} c_{i_{h} i_{k}} \cdot \exp \left(\sum_{j=1}^{m} \xi_{i_{j}}\right)
$$

for all $m$ such that $2 \leq m \leq N$, and is equal to 0 if $m>N$ (the reason for this is that, for $m>N$, in each term of the summation there is a factor of the form $c_{i i}=0$, for some $i$ ).

Step 3. Putting all this together, and using the series expansion of (2.4), we finally get the desired expression for the general $N$-soliton $\tau$-function:

$$
\begin{aligned}
\tau(x)=1+\sum_{i=1}^{N} a_{i} \exp \xi_{i} & +\sum_{1 \leq i<j \leq N} a_{i} a_{j} c_{i j} \exp \left(\xi_{i}+\xi_{j}\right)+\ldots \\
& =\sum_{\substack{0 \leq m \leq N \\
1 \leq i_{1}<\cdots<i_{m} \leq N}} \prod_{j=1}^{m} a_{i_{j}} \cdot \prod_{1 \leq h<k \leq m} c_{i_{h} i_{k}} \cdot \exp \left(\sum_{j=1}^{m} \xi_{i_{j}}\right) .
\end{aligned}
$$

\section{Group varieties of $N$-SOliton $\tau$-FunCtions}

In the preceding Section we have shown that the general $N$-soliton solution to the $\mathrm{KP}$ hierarchy is written as a particular linear combination of linear exponentials, with coefficients in $k$ (see $(2.5)$ ). This leads us to consider the following general situation:

$$
\tau(x)=\sum_{i=0}^{m} a_{i} \exp \left(b_{i} x\right) \in k[[x]]
$$

where $x=\left(x_{1}, \ldots, x_{n}\right), b_{i} x=\sum_{j=1}^{n} b_{i j} x_{j}$, and $k$ is an algebraically closed field of characteristic zero (not necessarily the complex field). 
It follows easily from the definition that this function is a holomorphic theta type, in fact it satisfies the holomorphic prosthaferesis (1.2):

$$
\begin{aligned}
\tau(x+y) \tau(x-y)= & {\left[\sum_{i=0}^{m} a_{i} \exp \left(b_{i} x+b_{i} y\right)\right] \cdot\left[\sum_{j=0}^{m} a_{j} \exp \left(b_{j} x-b_{j} y\right)\right]=} \\
& =\sum_{i, j} a_{i} a_{j} \exp \left(b_{i} x+b_{j} x\right) \exp \left(b_{i} y-b_{j} y\right) \in k[[x]] \otimes_{k} k[[y]]
\end{aligned}
$$

Let us denote by $C$ the hyperfield of the theta type $\tau(x)$, and by $A$ its group variety, i.e. $A$ is a commutative group variety over $k$ such that $C=k(A)$. Our purpose, in this section, is to give a precise description of the group variety associated to a theta type of the form (3.1), and, in particular, to apply this to the case of $N$-solitons (2.5).

We recall from Sect. 1 that $C$ is finitely generated over $k$ by the iterated logarithmic derivatives of $\tau(x)$, from the seconds on, and this implies that $C$ is a subfield of $K=k\left(\exp x_{1}, \ldots, \exp x_{n}\right)$. But we note that $K$ itself is a finitely generated hyperfield, with the same coproduct and inversion of $C$, which are induced by those defined on $k[[x]]$. Furthermore we have an isomorphism of hyperfields (i.e. an isomorphism of $k$-algebras preserving the coproduct and the inversion):

$$
\left(\begin{array}{c}
K=k\left(\exp x_{1}, \ldots, \exp x_{n}\right) \\
\text { with coproduct } \\
\mathbf{P}: x_{i} \mapsto x_{i} \otimes 1+1 \otimes x_{i} \\
\text { and inversion } \\
\rho: x_{i} \mapsto-x_{i}
\end{array}\right) \cong\left(\begin{array}{c}
K^{\prime}=k\left(y_{1}, \ldots, y_{n}\right) \\
\text { with coproduct } \\
\mathbf{P}: y_{i} \mapsto y_{i} \otimes y_{i} \\
\text { and inversion } \\
\rho: y_{i} \mapsto y_{i}^{-1}
\end{array}\right)
$$

Now let us make another brief digression in order to describe a natural model for the group variety of a finitely generated hyperfield.

If $C$ is a finitely generated hyperfield over $k$, we say that an automorphism $\sigma$ of $C$ over $k$ is left-invariant (resp. right-invariant) if it satisfies $(\sigma \otimes \iota) \mathbf{P}=\mathbf{P} \sigma$ (resp. $(\iota \otimes \sigma) \mathbf{P}=\mathbf{P} \sigma)$. When $C$ is cocommutative, these two conditions are equivalent and we simply say $\sigma$ is invariant. Let us denote by $G_{l}(C)$ (resp. $G_{r}(C)$ ) the set of left-invariant (resp. right-invariant) automorphisms of $C$ over $k$ : it is easy to prove that they are isomorphic subgroups of the group of all automorphisms of $C$ over $k$. The fundamental fact, proved in [6], is that this subgroup may be given the structure of an algebraic group, such that its function field is precisely the hyperfield $C$. In other terms, the set of (left-)invariant automorphisms of $C$ over $k$ may be regarded as the set of $k$-rational points of the group variety of $C$.

In our situation one sees immediately that the only invariant automorphisms of $K^{\prime}$ over $k$ are given by $\sigma\left(x_{i}\right)=a_{i} x_{i}$, for some $a_{i} \in k^{*}, i=1, \ldots, n$, so that we get an isomorphism $G_{l}\left(K^{\prime}\right) \cong\left(k^{*}\right)^{n}$, which shows that the group variety of $K^{\prime}$ (hence of $K)$ is $\left(k^{*}\right)^{n}$, the product of $n$ multiplicative groups. 
From what we have stated above, it follows that $C$ is a subhyperfield of $K$, hence we have a surjective homomorphism of group varieties $\left(k^{*}\right)^{n} \rightarrow A$, of the group variety of $K$ onto the one of $C$.

Now we recall a general result on algebraic groups (see, for example [5, Ch. 4, $\S 1$, Coroll. 2.4]), which states that every quotient of a multiplicative group is itself a multiplicative group. Hence we can conclude that $A$ must be isomorphic to $\left(k^{*}\right)^{s}$, for some $s \leq n$.

To complete the description of the variety $A$, there remains only the evaluation of its dimension $s$, which is equal to the transcendence degree of $C$ over $k$ (with regard to $C$, we can also note that it must be a pure transcendental extension of $k$, since its variety $\left(k^{*}\right)^{s}$ is rational).

In order to do that, let us recall from Section 1 that $\operatorname{transc}(C / k) \geq \operatorname{dim} \tau(x)$, so we now propose to evaluate the dimension of the theta type $\tau(x)$ : when $\tau(x)$ turns out to be non-degenerate, i.e. $\operatorname{dim} \tau(x)=n$, then $\operatorname{transc}(C / k)=n$ (it is always $\leq n$, from what we have stated before), hence $A=\left(k^{*}\right)^{n}$ and $C=K$.

We start by noting that we can always consider an associate theta type, obtained from the expression (3.1) by multiplication by the linear exponential $\exp \left(-b_{0} x\right)$; hence we are reduced to the case of functions of the form:

$$
\tau(x)=a_{0}+\sum_{i=1}^{m} a_{i} \exp \left(b_{i} x\right), \quad a_{i} \neq 0 \quad \forall i .
$$

Then, if the rank of the matrix of the $b_{i j}$ 's is equal to $r<n$, we can consider $y_{1}=b_{1} x, \ldots, y_{r}=b_{r} x$ as a new set of indeterminates (modulo a renumbering of the $b_{i}$ 's), and express the remaining $b_{r+1} x, \ldots, b_{m} x$ as linear combinations of the $y_{i}$ 's. With this change of variables we have an inclusion of $C$ in $k\left(\exp y_{1}, \ldots, \exp y_{r}\right)$, hence we have transc $(C / k) \leq r<n$ (actually the transcendence degree is equal to $r$, as we shall see later). This is the case in which $\tau(x)$ is degenerate, in fact it effectively depends on less than $n$ variables: it is not possible to reach the maximum dimension $n$ in this situation.

Therefore we are reduced to the case of a function $\tau(x)$ given by (3.3), with $m \geq n$ and $\operatorname{rank}\left(b_{i j}\right)=n$.

Proposition 3.4. - Under the preceding hypotheses, the theta type $\tau(x)$ is nondegenerate, i.e. $\operatorname{dim} \tau(x)=n$.

Proof. - If $\operatorname{dim} \tau(x)<n$, it follows that there exist a derivative $d=\sum_{i=1}^{n} \lambda_{i} d_{i}$, with $d_{i}=\partial / \partial x_{i}$ and $\lambda_{i} \in k$, and a theta type $\tau^{\prime}(x)$, associate to $\tau(x)$, such that $d \tau^{\prime}(x)=0$. Let $\phi(x)=\exp \left(\sum_{i=1}^{n} \beta_{i} x_{i}+\sum_{i, j} \gamma_{i j} x_{i} x_{j}\right)$ be a generic quadratic exponential, and $\tau^{\prime}(x)=\phi(x) \tau(x)$ a generic associate to $\tau(x)$. From $d \tau^{\prime}(x)=0$, it follows, by a straightforward computation, that:

$$
\left\{\begin{array}{l}
\sum_{s=1}^{n} \lambda_{s} \beta_{s}=0, \\
\sum_{s=1}^{n} \lambda_{s} \beta_{s}+\sum_{s=1}^{n} \lambda_{s} b_{i s}=0, \quad i=1, \ldots, m,
\end{array}\right.
$$


hence we must have $\sum_{s=1}^{n} \lambda_{s} b_{i s}=0 \forall i$, with $\lambda_{1}, \ldots, \lambda_{n}$ not all zero.

But this is in contrast with the hypothesis that $\operatorname{rank}\left(b_{i j}\right)=n$, hence $\tau(x)$ is non-degenerate. Q.E.D.

This result applies immediately to the case of solitons. Let us consider an $N$-soliton solution to the KP hierarchy, and suppose that it depends only on finitely many variables $x_{1}, \ldots, x_{n}$. From the end of Section 2 , we recall that it can be written as:

$$
\begin{aligned}
\tau(x)=1+a_{1} \exp \xi_{1}+\cdots+a_{N} \exp \xi_{N}+\cdots & +a_{i j} \exp \left(\xi_{i}+\xi_{j}\right)+\cdots+ \\
& +a_{12 \ldots N} \exp \left(\xi_{1}+\xi_{2}+\cdots+\xi_{N}\right),
\end{aligned}
$$

where $\xi_{i}=\xi\left(x, p_{i}\right)-\xi\left(x, q_{i}\right)=\sum_{j=1}^{n}\left(p_{i}^{j}-q_{i}^{j}\right) x_{j}$.

Using the notations of (3.3), this amounts to setting $b_{i j}=p_{i}^{j}-q_{i}^{j}$, for $i=1, \ldots, N$ and $j=1, \ldots, n$, while the $b_{i}$ 's, for $i>N$, are linear combinations of $b_{1}, \ldots, b_{N}$ (e.g., we have $b_{N+1}=b_{1}+b_{2}, b_{N+2}=b_{1}+b_{3}$, etc.).

The matrix of the $b_{i j}$ 's has therefore the following form:

$$
\left(\begin{array}{cccc}
p_{1}-q_{1} & p_{1}^{2}-q_{1}^{2} & \ldots & p_{1}^{n}-q_{1}^{n} \\
p_{2}-q_{2} & p_{2}^{2}-q_{2}^{2} & \ldots & p_{2}^{n}-q_{2}^{n} \\
\vdots & \vdots & & \vdots \\
p_{N}-q_{N} & p_{N}^{2}-q_{N}^{2} & \ldots & p_{N}^{n}-q_{N}^{n} \\
\hline \multicolumn{3}{c}{\text { other rows which are }} \\
\text { linear combinations of } \\
\text { the first } N \text { ones }
\end{array}\right)
$$

hence we can distinguish essentially two cases:

i) $n>N$ : for generic values of the parameters $p_{i}$ and $q_{j}$, the rank of (3.6) is equal to $N$, and it is not the highest possible. The $N$-soliton $\tau$-function (3.5) is a degenerate theta type and has $\left(k^{*}\right)^{N}$ as its associated group variety.

ii) $n \leq N$ : for generic values of the parameters $p_{i}$ and $q_{j}$, the rank of (3.6) is $n$. In this case the group variety related to $\tau(x)$ is $\left(k^{*}\right)^{n}$, and has the highest possible dimension. Correspondingly $\tau(x)$ is a non-degenerate theta type.

We have thus proved the following

THEOREM 3.7. - An N-soliton solution $\tau(x)$ to the KP hierarchy, depending on $n$ independent variables, is a holomorphic theta type. Its group variety is $\left(k^{*}\right)^{s}$, the direct product of $s$ copies of the multiplicative group $k^{*}$, where $s$ is generically equal to $\min (N, n)$.

As a final remark, we note that all this applies not only to soliton solutions of the KP hierarchy, but also to those of other hierarchies (such as KdV), which are specializations of the KP. 


\section{REFERENCES}

[1] I. Barsotti, Considerazioni sulle funzioni theta, Symp. Math., 3 (1970), p. 247.

[2] I. Barsotti, Le equazioni differenziali delle funzioni theta, Rend. Acc. Naz. XL, 101 (1983), p. 227.

[3] F. Bottacin, Group varieties related to the KP hierarchy, Ann. Mat. Pura Appl. (IV), Vol. CLXII (1992), p. 215.

[4] E. Date - M. Jimbo - M. Kashiwara - T. Miwa, Transformation groups for soliton equations, in Proceedings RIMS Symp. Nonlinear Integrable Systems-Classical Theory and Quantum Theory (Kyoto 1981), World Scientific, Singapore (1983), p. 39.

[5] M. Demazure - P. Gabriel, Groupes algébriques, tome I, Masson, North-Holland (1970).

[6] G. Gerotto, Alcuni elementi di teoria degli ipercorpi, Ann. Mat. Pura Appl., 115 (1977), p. 349 .

[7] D. Mumford, Tata Lectures on Theta, vol. 2, Birkhäuser, Boston-Basel-Stuttgart (1984).

[8] T. Shiota, Characterization of Jacobian varieties in terms of soliton equations, Invent. Math., 83 (1986), p. 333.

Dipartimento di Matematica Pura e Applicata, Università degli Studi di Padova,

Via Belzoni 7, 35131 Padova, Italy

E-mail address: bottacin@math.unipd.it 